\title{
Negative Chromatic Dispersion Generated by Introducing Curvature into Photonic Crystal Fiber
}

M. Lucki

This is a modeling work, which aims to show that negative chromatic dispersion (CD) may be obtained in a PCF by fiber bending. Results from the study of negative dispersion could be employed in a new dispersion compensating technique. The proposed method does not require doping in the core, and does not require external cores. The minimum negative dispersion achieved by this method was $-185000 \mathrm{ps} / \mathrm{nm} / \mathrm{km}$. Problems of bending losses and sensitivity of the dispersion with respect to deviations of geometry were studied.

Keywords: Negative chromatic dispersion, Photonic Crystal Fiber, bending radius, normalized hole diameter, bending losses.

\section{Negative dispersion in PGFs}

\subsection{Existing dispersion compensating techniques and methods for negative $C D$}

Negative CD could be used in telecommunications for opposite slope dispersion compensation, and can be achieved by various methods, such as those based on chirped gratings [1], dispersion compensating filters [2], Brewster-angled chirped mirrors [3], and soliton-induced negative dispersion [4]. The most mature dispersion compensating technique is the use of dispersion compensating fibers (DCFs) [5]. The first conventional DCF with a large index contrast and a small core exhibited a negative chromatic dispersion coefficient value of tens of $\mathrm{ps} / \mathrm{nm} / \mathrm{km}$ at $1550 \mathrm{~nm}$ [6]. More recent dual concentric core optical fibers, consisting of a single-mode central core surrounded by a guiding ring, achieve a minimum CD of $1800 \mathrm{ps} / \mathrm{nm} / \mathrm{km}$ at $1550 \mathrm{~nm}$ [7]. Most models proposed as PCF-based DCFs suggest the presence of dual concentric cores - by introducing one ring with smaller holes or removing an entire chosen ring of holes [8] [9], or creating proper index diversity by doping to ensure significant index contrast [10]. The essential problem of fibers with selective filling of the core, with a small core or with a great air-filling fraction is an often unacceptable loss, a small effective mode area or an unfitting operating wavelength range that disqualifies the fiber for high-speed transmission systems because of high attenuation at $1550 \mathrm{~nm}$. Increasing the index of a core can also lead to higher order modes that may interfere in a confining core with the fundamental mode and cause modal noise, as suggested in [8]. An MOF undoped in the core with seven dual cores designed for CD compensation was proposed by Zhang et al [8]; the negative dispersion coefficient was $4500 \mathrm{ps} / \mathrm{nm} \mathrm{km}$. Although this may result in thousands of $\mathrm{ps} / \mathrm{nm} / \mathrm{km}$ of negative dispersion, negative the CD was sensitive to hole diameter deviation. One of the highest ever-published negative CD coefficients ( $50000 \mathrm{ps} / \mathrm{nm} / \mathrm{km}$ in $\mathrm{PCF}$ and $-590000 \mathrm{ps} / \mathrm{nm} / \mathrm{km}$ in an MOF with a dual concentric core) was published by Yang et al in [9]. Negative CD in the case of fiber bending has been obtained only in a step-index fiber [12] (2007), where LP02 is not scattered.

\subsection{Possibilities of modeling a bent PCF with coupling modes}

Fiber bending with a small curvature radius is not trivial because the total internal reflection condition should be kept. Fiber bending could be sufficiently expressed by introducing curvature into the refractive index profile, represented by the curvature radius and the orientation in radians. The bend orientation defines the plane in which the waveguide is bent, and is referenced to the positive $\mathrm{x}$-axis in a clockwise fashion. A bend orientation of $0 \mathrm{rad}$ denotes that the waveguide is bent in the $x$-z plane, while a bend orientation of $\pi / 2$ denotes that it is bent in the $y-z$ plane. The results contained in this work refer to a bending orientation of $0 \mathrm{rad}$. The mechanism of bending of MOFs was analyzed by Eijkelenborg et al [12] in the sense observing the coloring the white pulse launched into the fiber with five cores. Bending-induced coloring can be described by a model, based on angular Bragg grating confinement. Another approach employs the theory of coupling modes. If the central core is single-mode at $1550 \mathrm{~nm}$ with electric field distribution $E_{1}(x, y)$ and effective index $n_{\text {effl }}$, then the cladding mode caused by the bend is described

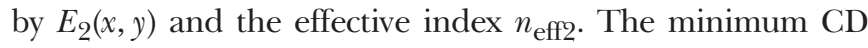
occurs at the wavelength for which the difference of two effective indices is zero, as predicted by Auguste et al [7]. This occurs at a certain wavelength, known as the phase-matching wavelength:

$$
n_{\text {eff1 }}-n_{\text {eff2 }}=\left|n_{\text {eff1 }}-n_{\text {eff2 }}\right|=0 .
$$

PCF bent at a curvature radius $R$ and an equivalent RIP, taking the curvature into account, can be expressed: 


$$
n_{2}(x, y)=n_{1}(x, y)\left(1+\frac{x}{R}\right)
$$

Where $n_{1}$ is the refractive index of the material characterized by position $(x, y)$ (before bending) and $n_{2}$ is the refractive index of the material at the same point, corrected by a coefficient taking into account the impact of bending by adjusting the $x$ position by the value of bending radius $R$, assuming bending orientation in the $x-z$ plane ( 0 radians from the $x$-axis), so that adjustment of the $y$ coordinate is not necessary. Such assumptions have been made, e.g., in Fevrier et al. [11]. Bent fiber theoretically propagates two supermodes: the fundamental LP01 mode leading to negative CD and the cladding mode (modes) leading to a very positive coefficient of CD. In the case of dual concentric core step-index fibers, the value of the negative $\mathrm{CD}$ coefficient was comparable to the positive one [13]. For theoretical assumptions, we consider two propagating supermodes: the central supermode LP01, guided in the central core surrounded by the microstructured cladding, assumed to be a single mode at $1550 \mathrm{~nm}$; the outer supermode, created by light coupled from the central-core mode, propagating in the silica glass background, and scattered on the air inclusions. The outer supermode can be multimode in the C-band as there is not enough space between the holes to form one mode or no possibility to form a cladding defect mode. The fiber is designed so that the fundamental modes of both guides couple at the phase-matching wavelength $\lambda_{0}$, providing a highly negative $\mathrm{CD}$ of the first supermode. Beyond the phase-matching wavelength, for certain curvature of the bending, coupling leads to intensity of the cladding mode greater than the intensity of LP01. In order to analyze the behavior of the complete structure, we make use of the coupled mode theory, as described in [14] and [15]. We consider the two supermodes as the elementary modes of the two independent guides. The electric fields of the fundamental modes of the structure (composed of the association of LP01 guided in the core and mode guided in the cladding), called supermodes, are then given by:

$$
\begin{aligned}
& \varepsilon_{I}=\psi_{I} \exp ^{-j \beta_{I} z}, \\
& \varepsilon_{I I}=\psi_{I I} \exp ^{-j \beta_{I I} z},
\end{aligned}
$$

where $\psi_{i}$ is the radial distribution, and $\beta_{i}$ is the propagation constant of the considered mode. To simplify the definition, we decompose the supermodes into elementary modes (or inversely) in a cross-section as:

$$
\begin{aligned}
& \psi_{I}=b_{I 1} \psi_{1}+b_{I 2} \psi_{2}, \\
& \psi_{I I}=b_{I I 1} \psi_{1}+b_{I I 2} \psi_{2} .
\end{aligned}
$$

In this relation, the modal coefficient is defined as:

$$
b_{I 1}=\frac{\frac{\beta_{1}}{2 \mu_{0} \omega} 2 \pi \int_{0}^{\infty} \psi_{I} \psi_{1} r \mathrm{~d} r}{P_{m 1}=1}=\frac{\int_{0}^{\infty} \psi_{I} \psi_{1} r \mathrm{~d} r}{\int_{0}^{\infty} \psi_{1}^{2} r \mathrm{~d} r} .
$$

In the same way, we can obtain the $b_{I 2}, b_{I I 1}$, and $b_{I I 2}$ coefficients. The amplitudes of the field of the first supermode and the two elementary modes are positive at all points in the cross section. The second supermode is chosen to be positive in the axis, so $b_{I 1}, b_{I 2}, b_{I I 1}$ must be positive and $b_{I I 2}$ negative. These different coefficients satisfy (at any wavelength) equations given by the coupled mode theory. At the phase-matching wavelength $\lambda_{0}$, the modal coefficients become:

$$
b_{I 1}=b_{I 2}=b_{I I 1}=-b_{I I 2} \text {. }
$$

Indeed, at $\lambda_{0}$, the power of the two supermodes is distributed in the two cores, and the presence of one will gradually excite the other automatically for the same effective index of both guides. The radial distribution of the modes can be obtained accurately by a numeric resolution. The full-vectorial FDFD technique with index averaging and the modified Yee's algorithm was used to obtain the results contained in this paper [16]. The constant of propagation can be obtained by the formula:

$$
\beta=\frac{2 \pi n_{\mathrm{eff}}}{\lambda_{0}}[1 / \mathrm{m}] .
$$

After substitution for group velocity and group delay, we may derive the dispersion by the formula:

$$
D=\frac{\mathrm{d} \tau}{\mathrm{d} \lambda}[\mathrm{ps} /(\mathrm{nm} \mathrm{km})]
$$

We solve the Maxwell equations describing the propagation launched in the $z$ axis, and, after discretization and applying mesh algorithm, we obtain matrices that are solved numerically, followed by the algebra leading to eigenvalue equations in terms of transverse fields:

$$
i k_{0}\left[\begin{array}{l}
H_{x} \\
H_{y} \\
H_{z}
\end{array}\right]=\left[\begin{array}{ccc}
0 & -i \beta I & U_{y} \\
i \beta I & 0 & -U_{x} \\
-U_{y} & U_{x} & 0
\end{array}\right] \cdot\left[\begin{array}{c}
E_{x} \\
E_{y} \\
E_{z}
\end{array}\right],
$$

$$
-i k_{0}\left[\begin{array}{ccc}
\varepsilon_{r x} & 0 & 0 \\
0 & \varepsilon_{r y} & 0 \\
0 & 0 & \varepsilon_{r z}
\end{array}\right] \cdot\left[\begin{array}{l}
E_{x} \\
E_{y} \\
E_{z}
\end{array}\right]=\left[\begin{array}{ccc}
0 & -i \beta I & V_{y} \\
i \beta I & 0 & -V_{x} \\
-V_{y} & V_{x} & 0
\end{array}\right] \cdot\left[\begin{array}{l}
H_{x} \\
H_{y} \\
H_{z}
\end{array}\right] .
$$

After estimations and trial simulations, as the curvature radius of about $66000 \mu \mathrm{m}$ may satisfy the condition for total internal reflection, initial curvature radius $R$ was set to $66000 \mu \mathrm{m}$. Then it was possible to adjust the geometry to receive the negative $\mathrm{CD}$ in the $\mathrm{C}$-band.

\subsection{The sensitivity of minimum negative CD to deviations of the curvature radius or with respect to the normalized hole diameter}

During the fabrication process, the predicted values of the hole diameter or the curvature radius could have some deviations from the theoretical predictions. The holes could have a 
different radius (if the air pressure during the drawing process is not suitable). The prediction of CD for deviation of the curvature radius or the normalized hole diameter deviation is presented here. An optimum reference configuration resulting in minimum negative $\mathrm{CD}$ in the $\mathrm{C}$-band was predicted, as follows: pitch $\Lambda=23.2 \mu \mathrm{m}, \mathrm{d} / \Lambda=0.50453$ (for investigation of the curvature radius deviation), and pitch $\Lambda=23.2 \mu \mathrm{m}$, bending radius $R=66116 \mu \mathrm{m}$ (for the investigation of the hole diameter deviation).

\section{Numerical results}
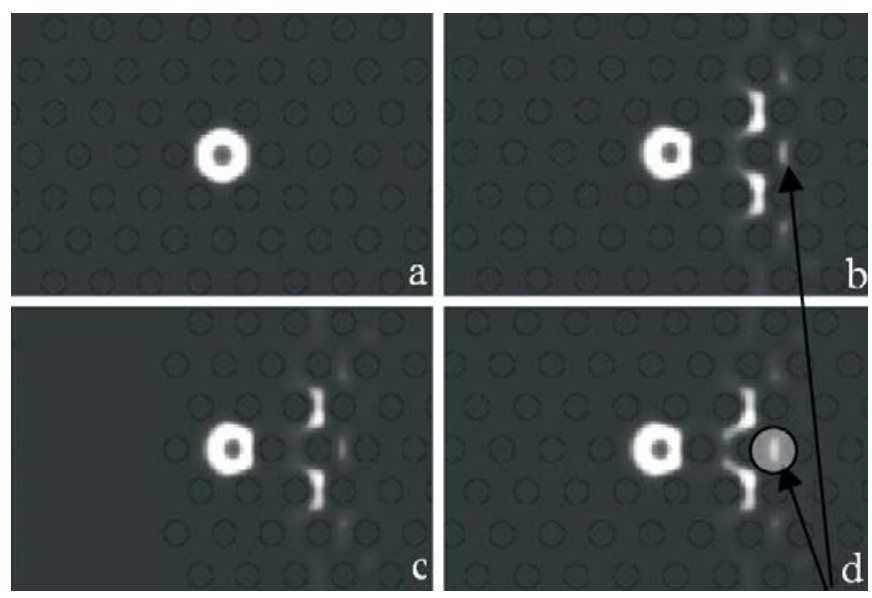

Fig. 1: Fundamental mode before introducing curvature (a) coupling from LP01 to the cladding modes (b) coupling interaction untouched for imprecision or absence of holes in the $x<0$ plane (c) weaker nonlinearity for even small imprecision of a hole in the $x>0$ plane with strong confinement of the optical power

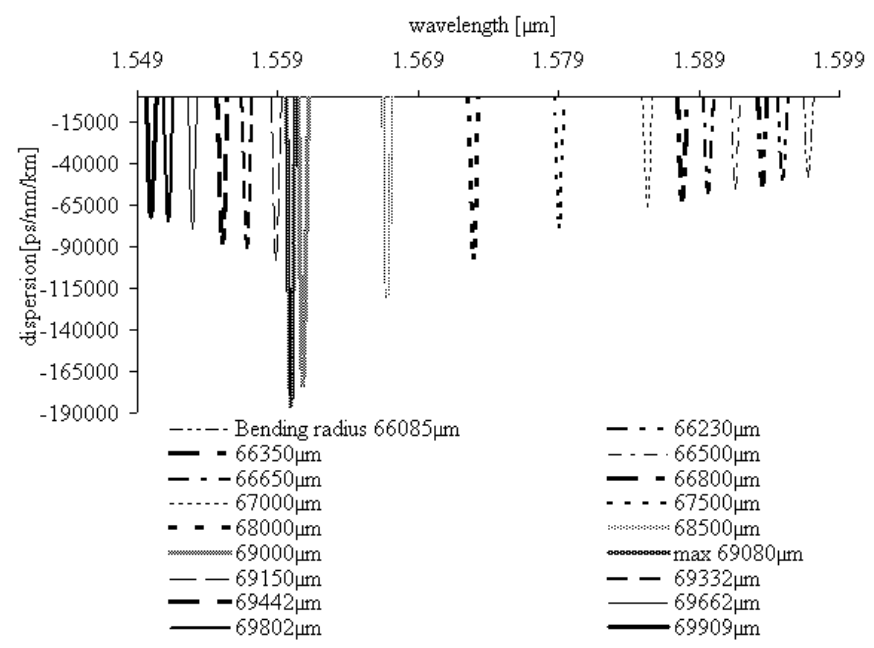

Fig. 2: Minimum negative $\mathrm{CD}$ with respect to different bending radii for assumed $\mathrm{d} / \Lambda=0.50453$

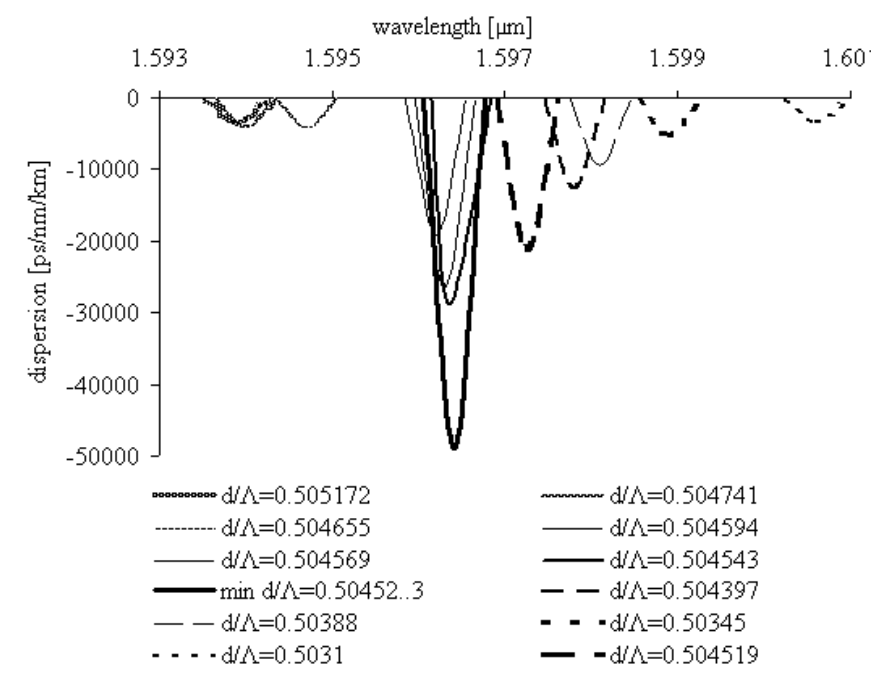

Fig. 3: Minimum negative dispersion with respect to deviation of the hole diameter for assumed bending at radius $66115.7 \mu \mathrm{m}$

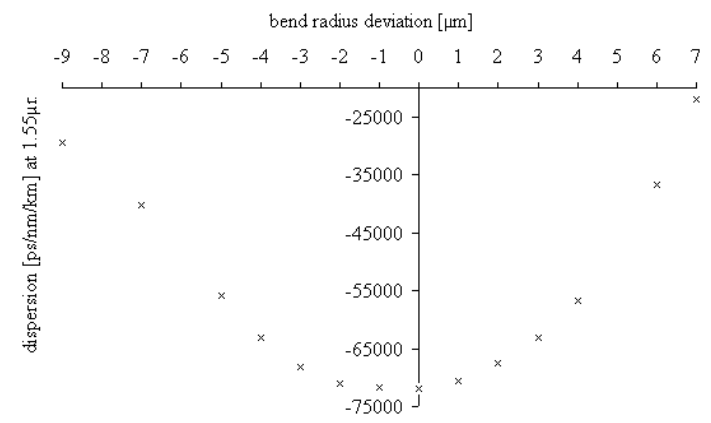

Fig. 4: Value of negative chromatic dispersion at exactly $1550 \mathrm{~nm}$ for different bending radii deviations

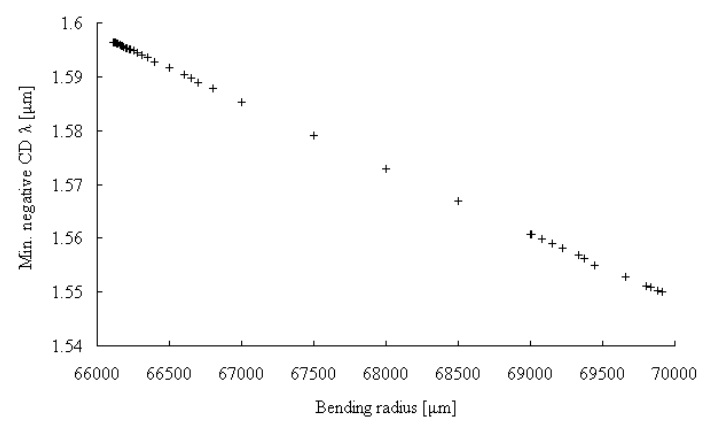

Fig. 5: Linear evolution of the minimum negative CD-wavelength as a function of bending radius

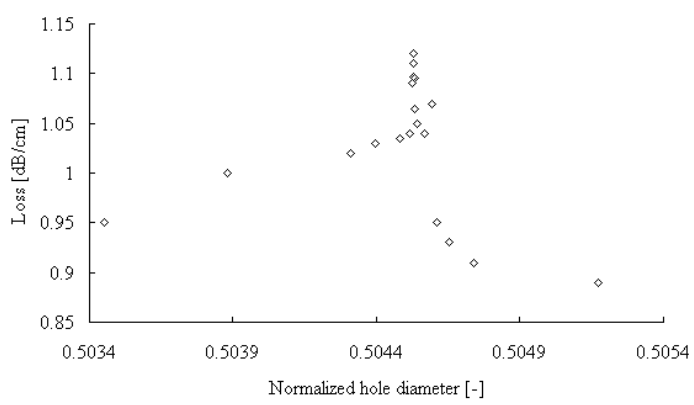

Fig. 6: Bending losses as a function of normalized hole diameter for assumed curvature radius $66116 \mu \mathrm{m}$ 


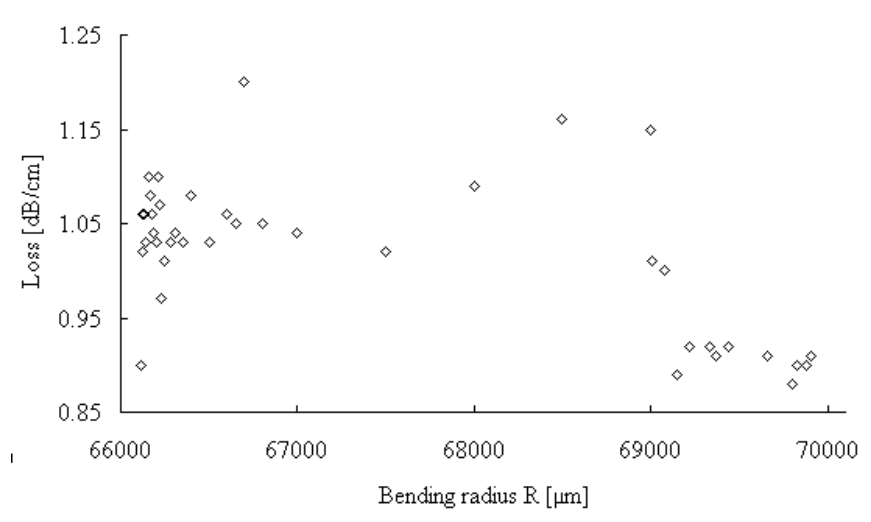

Fig. 7: Bending losses as a function of curvature radius for assumed normalized hole diameter $\mathrm{d} / \Lambda=0.504530$

\section{Discussion}

Negative chromatic dispersion (CD) can be generated by fiber bending, resulting from a bending-induced mechanism of coupling from the fundamental mode to the cladding modes. The core guide and the cladding guide reflect light by the same effective index, and coupling is possible when both supermodes propagate with the same velocity, at a specific phase-matching wavelength [Fig. 1b], and resulting in strong optical nonlinearities, i.e., highly nonlinear wavelength dependency of chromatic dispersion with significant negative CD over a short wavelength range. The zero-dispersion point is halfway between the negative and positive CD peak (e.g., if minimum negative $\mathrm{CD}$ was at $1.55 \mu \mathrm{m}$, maximum positive $\mathrm{CD}$ was at $1.5502 \mu \mathrm{m}$, and the zero-dispersion wavelength was $1.5501 \mu \mathrm{m})$. The character of the effective index-matching tangent is different for a different fiber geometry. We obtained the biggest minimum negative CD for normalized hole diameter 0.50453 for a PCF bent at a radius of $69080 \mu \mathrm{m}$ at $1559.9 \mathrm{~nm}$. The negative CD was $-185420 \mathrm{ps} / \mathrm{nm} / \mathrm{km}$.

An investigation of the behavior of minimum negative CD with respect to the curvature radius showed that a smaller curvature radius (stronger bending) is responsible for tuning a phase-matching wavelength towards longer wavelengths [Fig. 2]. The value of the minimum negative-CD wavelength is sharply sensitive to the bending radius, and the bandwidth of the negative $\mathrm{CD}$ is not flat. Changing the curvature radius by $100 \mu \mathrm{m}$ shifts the negative dispersion peak by $0.01 \mu \mathrm{m}$ and influences the minimum CD. When the curvature radius was varied by $580 \mu \mathrm{m}$ from $69080 \mu \mathrm{m}$ to $68500 \mathrm{~mm}(0.84 \%$ of the reference curvature radius, a smaller curvature radius means greater bending), the dispersion peak was shifted by about $6.9 \mathrm{~nm}$ into longer wavelengths, and the value of the extreme decreased by $35 \%$ from $-185420 \mathrm{ps} / \mathrm{nm} / \mathrm{km}$ to $-119610 \mathrm{ps} / \mathrm{nm} / \mathrm{km}$. When the curvature radius had the equivalent positive deviation, the dispersion peak was shifted by $7 \mathrm{~nm}$ into shorter wavelengths, and the value of the CD extreme decreased by $57 \%$, from $-185420 \mathrm{ps} / \mathrm{nm} / \mathrm{km}$ to $-79185 \mathrm{ps} / \mathrm{nm} / \mathrm{km}$. However, half-decreased minimum negative $\mathrm{CD}$ is over a wide range of curvature radii (few centimeters). The minimum negative CD wavelength exhibited a linear dependency on the bending radius, as depicted in [Fig. 5]. Similar behavior would refer to the zero-dispersion wavelength, which was slightly longer than the minimum negative CD wavelength. Summarizing, a negative tolerance to the radius of curvature is preferred (smaller curvature radius or a greater bend).

The negative CD peak exhibited a shift towards shorter wavelengths, and its value demonstrated little variation when increasing the hole diameter. Whereas the same dispersion peak was shifted towards longer wavelengths, the value of the peak demonstrated a remarkable variation, and became less negative when the hole diameter was decreased [Fig. 3]. A certain negative tolerance to the hole diameter is preferred (smaller holes). The value was decreased by about $74 \%$ for a certain negative deviation, and by $90 \%$ for an equivalent positive deviation. When the hole diameter was increased by 0.000082 , the position of the dispersion peak was shifted by $1.5 \mathrm{~nm}$ towards shorter wavelengths, and the value of the peak was decreased by $90 \%$ from $-48850 \mathrm{ps} / \mathrm{nm} / \mathrm{km}$ to $-4570 \mathrm{ps} / \mathrm{nm} / \mathrm{km}$. When $\mathrm{d} / \Lambda$ had an equivalent positive deviation, the position of the dispersion peak was shifted by about $1.5 \mathrm{~nm}$ towards a longer wavelength, and the peak's value was decreased by $74 \%$ from $-48850 \mathrm{ps} / \mathrm{nm} / \mathrm{km}$ to $-12643 \mathrm{ps} / \mathrm{nm} / \mathrm{km}$. For greater imprecision, the question, if it should be negative or positive tolerance is unimportant. When the diameter of the holes was increased by 0.000642 , the position of minimum CD was shifted by about $2.5 \mathrm{~nm}$ towards shorter wavelengths and the minimum negative $\mathrm{CD}$ decreased by $90 \%$ from $-48850 \mathrm{ps} / \mathrm{nm} / \mathrm{km}$ to $-3303 \mathrm{ps} / \mathrm{nm} / \mathrm{km}$.

The hole diameter predicted to give the biggest possible minimum negative CD for given bending (and at determined wavelength) is not optimal for other curvature radii. As the minimum negative CD of a PCF bent at radius $69080 \mu \mathrm{m}$ occurred at $1559.9 \mathrm{~nm}$ for $\mathrm{d} / \Lambda=0.50453$, and we manipulated the curvature radius to tune the minimum negative $\mathrm{CD}$ into a $-1550 \mathrm{~nm}$ wavelength, the normalized hole diameter had to be adjusted, too. The curvature radius is then the main factor for tuning the minimum negative $\mathrm{CD}$ wavelength, while a proper hole diameter scales the minimum negative $\mathrm{CD}$ and weakly tunes the operating wavelength at the assumed bending. If the goal is to achieve the biggest possible negative $\mathrm{CD}$ at an arbitrary wavelength, it is rational to assume smaller tolerance to $\mathrm{d} / \Lambda$. If the aim is to precisely tune a possible negative $\mathrm{CD}$ wavelength, it is appropriate to assume smaller tolerance to the bending radius.

A cladding defect in the plane of coupling is considered as a deviation of the RIP, as it was responsible for a reduction of interesting optical nonlinearities [Fig. 1d]. We introduced negative deviation to one hole that is in the region with propagating light (radius $=5.7 \mathrm{~mm}$ instead of $5.85255 \mu \mathrm{m}$ ). As a result, $\mathrm{CD}$ at $1.55 \mu \mathrm{m}$ was decreased by $15524 \mathrm{ps} / \mathrm{nm} / \mathrm{km}$. The disturbance of the regularity of the structure (even a small imprecision of certain holes only) in the plane with coupled power could damage the existing negative $\mathrm{CD}$, while any imprecision of the holes in the plane of $x<0$ (opposite to the bending orientation) exhibits no impact on the negative CD [Fig. 1c]. An important question concerns not the value of an extreme, but the value at $1.55 \mu \mathrm{m}$. Attenuation of about $20000 \mathrm{ps} / \mathrm{nm} / \mathrm{km}$ at $1.55 \mu \mathrm{m}$ corresponds to a deviation of the curvature radius of $5 \mu \mathrm{m}$ [Fig. 4]. Sensitivity to bend radius deviation is less drastic for greater deviations.

Increased loss is for wavelengths characterized precisely by significant negative $\mathrm{CD}$, and the maximum losses are exactly for minimum negative CD. the bending loss is then 
very sensitive to hole diameter adjustments [Fig. 6]. For minimum negative CD of $-185420 \mathrm{ps} / \mathrm{nm} / \mathrm{km}$, the bending loss was about $1 \mathrm{~dB} / \mathrm{cm}$. On the other hand, as the adjustment of the curvature radius tunes the minimum negative-CD wavelength, the bending loss as a function of curvature radius $R$ exhibited similar wavelength behavior, but the value of the loss for minimum negative $\mathrm{CD}$ displayed no exact regularity and oscillated around $1 \mathrm{~dB} / \mathrm{cm}$ [Fig. 7]. The effect of coupling between modes on bending losses in PCFs was investigated by Olszewski et al in [17]. They demonstrated that the coupling between the fundamental mode and the gallery of the cladding modes causes oscillations in the dependence of the bending losses on $R$ within the short wavelength bending loss edge in large core PCFs.

\section{Conclusions}

The method proposed in this paper is based on introducing curvature into the fiber, which results in negative dispersion at a certain wavelength. The highest value achieved by this method was $-185000 \mathrm{ps} / \mathrm{nm} / \mathrm{km}$ in a microstructured fiber. Negative CD was the most sensitive to hole diameter deviation, especially in the area of strong confinement of the optical power. The position of a peak is sharply sensitive to the curvature radius. A certain negative tolerance is preferred to both hole diameter deviation and radius of curvature. The disadvantage of this method are a significant loss around $1 \mathrm{~dB} / \mathrm{cm}$ from light scattered on the air holes, and the necessity to make corrections to the mode profile to match the Numerical Aperture of the compensating and compensated fiber, which can be done by a lens. It is not necessary to have a kilometer-long fiber, e.g., CD equal to $375 \mathrm{ps} / \mathrm{nm}$ of the $250-\mathrm{km}$-long link (the distance from Prague to Brno), assuming small dispersion of a conventional IGPCF, as $1.5 \mathrm{ps} / \mathrm{nm} / \mathrm{km}$ can be compensated with two metres of the proposed compensating fiber, if only it has negative dispersion $-185000 \mathrm{ps} / \mathrm{nm} / \mathrm{km}$. Advantages of the proposed method are not only record-breaking dispersion, but also the large effective mode area of the dispersive fundamental mode $\left(700 \mu \mathrm{m}^{2}\right)$. This is more than ten times that of the fiber proposed in [8], and seventeen times more than in [9], achieved in a DCF with external cores. Finally, the zero-dispersion wavelength can be tuned by winding a bending-based DCF onto a reel over tens of nanometers; the method does not require doping in the core. Bending-induced negative CD would be suitable for compensating the dispersion in high-speed transmission systems after reducing the bending losses and correcting the mode profile.

\section{References}

[1] Hill, O., Bilodeau, F., Halo, B., Kitagawa, T., Theriault, S., Johnson, C., Albert, J., Takiguchi, K.: Chirped in-Fiber Bragg Gratings for Compensation of Optical-Fiber Dispersion, Opt. Lett. Vol. 19 (1994), p. 1314-1316.

[2] Takiguchi, K., Okamoto, K., Moriwaki, K.: Planar Lightwave Circuit Dispersion Equalizer, J. Lightwave Technol. Vol. 14 (1996), p. 2003-2011.

[3] Steinmeyer, G.: Brewster-Angled Chirped Mirrors for High-Fidelity Dispersion Compensation and Bandwidths Exceeding One Optical Octave, Opt. Express, Vol. 11 (2003), No. 19.
[4] Sakamaki, K., Nakao, M.: Soliton Induced Supercontinuum Generation in Photonic Crystal Fiber, IEEE J. of Selected Topics in Quantum Electronics, Vol. 10 (2004), No. 5, September/October 2004.

[5] Gruner-Nielsen, L., Knudsen, S., Edvold, B., Veng, T., Magnussen, D., Larsen, C., Damsgaard, H.: Dispersion Compensating Fibers, Opt. Fiber Technol. Vol. 6 (2000), p. $164-80$.

[6] Antos, A., Smith, D.: Design and Characterization of Dispersion Compensating Fiber Based on the LP01 Mode, J. Lightw. Technol., Vol. 12 (1994), No. 10, p. 1739-1745.

[7] Auguste, J. et al.: Invited Paper Optical Fiber Technology, Vol. 8 (2002), p. 89.

[8] Zhang, Y., Yang, S., Peng, X., Lu, Y., Chen, X., Xie, S.: Design of Large Effective Area Microstructured Optical Fiber for Dispersion Compensation, In: Photonic Crystals and Fibers. Bellingham: Spie, Vol. 5950 (2005), No. 43, ISBN 0-8194-5957-7.

[9] Yang, S., Zhang, Y., Peng, X., Lu, Y., Xie, S., Li, J., Chen, W., Jiang, Z., Peng, J., Li, H.: Theoretical Study and Experimental Production of High Negative Dispersion Photonic Crystal Fiber with Large Area Mode Field, Opt. Express 3015, Vol. 14 (2006), No. 7.

[10] Zsigri, B., L gsgaard, J., Bjarklev, A.: A Novel Photonic Crystal Fibre Design for Dispersion Compensation, J. Opt. A: Pure Appl. Opt. Vol. 6 (2004), p. 717-720.

[11] Fevrier, S., Auguste, J.-L., Blondy, J.-M., Peyrilloux, A., Roy, P., Pagnoux, D.: Accurate Tuning of the Highly-Negative Chromatic Dispersion Wavelength into a Dual Concentric Core Fibre by Macro-Bending, Fibers and Waveguide Components P1.8, 2007.

[12] Eijkelenborg, M., Canning, J., Ryan, T.: Bending-Induced Colouring in a Photonic Crystal Fibre, Opt. Express 88, Vol. 7 (2000), No. 2.

[13] Gerome, F., Auguste, J., Maury, J., Blondy, J., Marcou, J.: Theoretical and Experimental Analysis of a Chromatic Dispersion Compensating Module Using a Dual Concentric Core Fiber, J. Lightwave Technol. Vol. 24 (2006), No. 1.

[14] Cozens, J. R., Boucouvalas, A. C.: Coaxial Optical Coupler, Electron. Lett., Vol. 18 (1982), No. 3, p. 138-140, Jul. 1982

[15] Boucouvalas, A. C.: Coaxial Optical Fiber Coupling, J. Lightwave Technol., Vol. LT-3 (1985), No. 5, p. 1151-1158, Oct. 1985

[16] Zhu, Z., Brown, T. G.: Full-Vectorial Finite-Difference Analysis of Microstructured Optical Fibers, Optics Express 853 , Vol. 10 (2002), No. 17.

[17] Olszewski, J., Szpulak, M., Urbanczyk, W.: Effect of Coupling Between Fundamental and Cladding Modes on Bending Losses in Photonic Crystal Fibers, Opt. Express 6015, Vol. 13 (2005), No. 16.

Michal Lucki

luckim1@fel.cvut.cz

Dept. of Telecommunication Engineering

Czech Technical University in Prague

Faculty of Electrical Engineering

Technická 2

16627 Prague, Czech Republic 